\title{
The Accelerating Universe and a Limiting Curvature Proposal
}

\author{
Damien A. Easson \\ Centre for Particle Theory, Department of Mathematical Sciences, Durham \\ University, Science Laboratories, South Road, Durham, DH1 3LE, U. K. \\ E-mail: damien.easson@durham.ac.uk
}

\begin{abstract}
We consider the hypothesis of a limiting minimal curvature in gravity as a way to construct a class of theories exhibiting late-time cosmic acceleration. Guided by the minimal curvature conjecture (MCC) we are naturally lead to a set of scalar tensor theories in which the scalar is non-minimally coupled both to gravity and to the matter Lagrangian. The model is compared to the Lambda Cold Dark Matter concordance model and to the observational data using the "gold" SNeIa sample of Riess et. al. (2004). An excellent fit to the data is achieved. We present a toy model designed to demonstrate that such a new, possibly fundamental, principle may be responsible for the recent period of cosmological acceleration. Observational constraints remain to be imposed on these models.

PACS numbers: 98.80.-k, 04.50.+h, 95.36.+x

arXiv: astro-ph/0608034
\end{abstract}

DCPT-06/17 


\section{Introduction}

One of the most profound discoveries of observational physics is that the universe is accelerating in its expansion [1, 2, 3, 4, 5, 6]. There have been many attempts to explain this late-time acceleration, for example, a pure cosmological constant, dark energy associated with some new scalar field and modified gravitational theories, although all current models require some level of fine-tuning and none are considered to be a complete explanation $\ddagger$. Whatever is responsible for the current acceleration may arise from some completely new physical principle. This is the possibility we consider in this paper. Our goal is to construct a toy model that represents a late-time accelerating Universe using a new, possibly fundamental, principle. As our guiding principle, we hypothesize the existence of a minimal curvature scale in gravity.

In a Friedmann, Robertson-Walker (FRW) space-time, without cosmological constant $\Lambda$ and with only standard matter sources such as dust and radiation, the universe will always decelerate as it expands. One way to avoid this is to add matter to the system that violates the strong energy condition (SEC). In a cosmological context this violation constitutes the addition of matter sources satisfying the equation of state $p \leq-1 / 3 \rho$. A second possibility is to explicitly remove flat space-time as a solution to the theory. In this case the vacuum of the theory, which is approached at late times as the energy density in matter fields becomes more and more dilute, is not Minkowski space-time, but instead an accelerating Universe [9, 10, 11]. To remove flat spacetime as a solution we hypothesize the existence of a minimal curvature in our underlying fundamental theory. The simplest example of this is, of course, to introduce a bare cosmological constant into General Relativity. However, in principle there may exist many other ways to achieve this result. Indeed, it appears that many accelerating cosmological models derived from modified gravity theories contain such a minimal curvature [12, 13, 14].

The idea of a minimal curvature scale in gravity mirrors that of a maximal curvature scale. In the literature many authors have considered this possibility and used it to remove the curvature singularities of General Relativity by bounding curvature invariants from above at the level of the classical action [15]-[26]. In the case of singularity removal, it is necessary to bound all curvature invariants in order to cover all possible physical situations in which such a singularity may occur.

By contrast, in the case of a minimal curvature approached at late times in a homogeneous, isotropic universe, symmetry implies that it is only necessary to bound the Ricci scalar $R$ from below. Hence, unlike in the case of a maximal curvature hypothesis, we shall see that one may implement a minimal curvature by using a modified Brans-Dicke theory where the Brans-Dicke field couples non-minimally to the matter Lagrangian.

Within this context we demonstrate that the existence of the minimal curvature (MC) produces a Universe that evolves from a matter dominated period to an $\ddagger$ For a recent reviews on the subject see [7, 8]. 
accelerating phase mimicking the $\Lambda$-Cold-Dark-Matter $(\Lambda \mathrm{CDM})$ model. We emphasize that the model presented here is only a toy construction of the late Universe. The model is not intended to provide a consistent cosmology from the time of Big-Bang Nucleosynthesis (BBN) until today. It is unlikely that the precise model presented here is compatible with solar system experiments and the tight constraints on the time variation of Newton's constant. However, the model does provide an example of how the postulated existence of a minimal curvature scale in gravity can provide a new mechanism to generate cosmological acceleration of the late Universe. Furthermore, the model may capture features of a possibly more fundamental theory that admits a minimal curvature scale $\mathcal{S}$.

In Section 2, we describe the minimal curvature construction, first by using a toy example and then by using a class of modified Brans-Dicke theories. We solve the equations of motion for this example and demonstrate how the Universe evolves from a matter dominated phase to an accelerating period as the curvature approaches its minimal value. In Section 3, we compare the MC model with $\Lambda$ CDM and to the supernovae (SNeIa) "gold" sample of [27]. Finally, we comment on the possibility of constructing more realistic models that satisfy the limiting curvature hypothesis and offer our conclusions and speculations in Section 4. In Appendix A, we provide a detailed analysis of the vacuum MC theory. In Appendix B, we construct an Einstein frame description of the vacuum theory and compare it to the $\mathrm{MC}$ vacuum.

\section{The Minimal Curvature Construction}

Our goal is to construct theories in which a certain physical quantity is bounded from below. Before leaping directly into our model, it is instructive to consider an example of how a similar effect may be achieved in a simpler theory - the bounding of velocities from above in Special Relativity by the speed of light [20].

The Newtonian action for a free particle of mass $m$ in motion is

$$
S=\int d t \frac{1}{2} m \dot{x}^{2} .
$$

In this classical theory the velocity of the particle is without bound. Now let us implement one of the fundamental consequences of Special Relativity: To ensure that the speed of this particle is limited by the speed of light we introduce a field $\phi(t)$ which couples to the quantity in the action that we want to bound $\left(\dot{x}^{2}\right)$ and has a "potential" $U(\phi)$. The resulting action is

$$
S=m \int d t\left[\frac{1}{2} \dot{x}^{2}+\phi \dot{x}^{2}-U(\phi)\right] .
$$

The variational equation with respect to $\phi$

$$
\dot{x}^{2}=\frac{\partial U}{\partial \phi}
$$

$\mathcal{S}$ An interesting example of a minimal curvature scale occurs in a certain classical limit of quantum gravity [13, 14]. 
ensures that $\dot{x}$ is bounded, provided $\partial U / \partial \phi$ is bounded. Note the absence of a kinetic term for $\varphi$ in the action, and hence, the reason the word potential appears in quotes above. In order to obtain the correct Newtonian limit for small $\dot{x}$ and small $\phi$ we take $U(\phi)$ proportional to $\phi^{2}$. In the Newtonian limit the action (2) reduces to (11). A simple potential satisfying the above asymptotics is

$$
U(\phi)=\frac{2 \phi^{2}}{1+2 \phi}
$$

Integrating out $\phi$ yields (up to an irrelevant constant) the action for relativistic particle motion:

$$
S_{S R}=m \int d t \sqrt{1-\dot{x}^{2}}
$$

The above model provides a powerful example of how a toy construction based on a fundamental principle - the existence of a universal "speed limit" - can capture features of a more fundamental theory.

We now use a similar construction to model the existence of a minimal curvature (MC) scale in gravity. Because we are interested in late time cosmology, we need only be concerned with bounding one curvature invariant, the Ricci scalar $R$. In direct analogy with our example from Special Relativity, we introduce a scalar field $\varphi$ that couples to the quantity we wish to bound, $R$, and a "potential" function $V(\varphi)$

$$
S_{M C}=\int d^{4} x \sqrt{-g}\left(\frac{M_{\mathrm{pl}}^{2}}{2} R-\gamma \varphi R+V(\varphi)\right),
$$

where $M_{\mathrm{pl}} \equiv(8 \pi G)^{-1 / 2}$ is the reduced Planck mass and both $\varphi$ and $\gamma$ posses dimensions of mass, $[\varphi]=[\gamma]=M$. The vacuum theory is equivalent to a Brans-Dicke theory with Brans-Dicke parameter $\omega_{B D}=0$. This is seen by re-writing the action (6) in terms of a new field

$$
\Phi=\left(\frac{M_{\mathrm{pl}}^{2}}{2}-\gamma \varphi\right)
$$

so that the action becomes

$$
S_{B D}=\int d^{4} x \sqrt{-g}(\Phi R+V(\Phi)) .
$$

Ordinarily, such a theory can be re-cast as a purely gravitational theory with Lagrangian $\mathcal{L}=\sqrt{-g} f(R)$ (see, e.g. [29]); however, this is not possible for all forms of the potential $V$.

For reasons that will become clear shortly, we allow the field $\varphi$ to couple nonminimally to matter. The non-minimal coupling yields the matter action,

$$
S_{M}=\int d^{4} x \sqrt{-g} \mathcal{L}_{M}\left(\psi_{i}, \varphi, f\left(\varphi / M_{p l}\right) \bar{\psi} \psi\right),
$$

where $\mathcal{L}_{M}$ is the Lagrangian made up of whatever matter fields $\psi_{i}$ are in the theory. In the case were $\psi$ represents a dark matter Dirac spinor, the field $\varphi$ couples non-minimally only to the dark matter sector and need not couple to baryons. In this case it is possible to avoid constraints on such a coupling from solar-system and table-top tests of gravity. 
In string theory, non-perturbative string loop effects do not generically lead to universal couplings, allowing the possibility that the dilaton decouples more slowly from dark matter than ordinary matter (see, e.g. [30, 31, 32, 33, 34, 35, 36]).

This coupling can be used to address the coincidence problem, since the acceleration is triggered by the coupling to matter. For the purposes of our toy construction, we do not distinguish between baryonic and dark matter in the remainder of our discussion.

Note that in the case of $f(R)$ theories which are conformally identical to models of quintessence in which matter is coupled to dark energy with a large coupling, this strong coupling induces a cosmological evolution radically different from standard cosmology [37]. Similar difficulties may arise in the model presented here, however, we have yet to investigate this issue.

The matter stress-energy tensor is given by

$$
T_{\mu \nu} \equiv-\frac{2}{\sqrt{-g}} \frac{\delta \mathcal{L}_{M}\left(\psi_{i}, \varphi\right)}{\delta g^{\mu \nu}} .
$$

We assume a perfect fluid

$$
T_{\mu \nu}=\left(\rho_{M}+P_{M}\right) u_{\mu} u_{\nu}+P_{M} g_{\mu \nu}
$$

where $u^{\alpha}$ is the fluid rest-frame four-velocity, and the energy density $\rho_{M}$ and pressure $P_{M}$ are related by the equation of state $P_{M}=w \rho_{M}$. Because we are focusing on the late Universe we shall ignore the presence of radiation and consider only a matter density $\rho_{M}$, which redshifts with expansion in the usual manner (with the exception of the non-trivial $\varphi$ dependence)

$$
\rho_{M} \propto \frac{f\left(\varphi / M_{\mathrm{pl}}\right)}{a^{3}} .
$$

In the above, $f$ is a function describing the non-minimal coupling of the field $\varphi$ to the matter Lagrangian. Such couplings in the context of ordinary scalar-Einstein gravity were studied in [36] where it was found that this coupling can be made consistent with all known current observations, the tightest constraint coming from estimates of the matter density at various redshifts. This coupling plays a critical role in Modified Source Gravity, introduced in [38].

Variation of the total action $S_{t o t}=S_{M C}+S_{M}$ with respect to the metric tensor, $\delta S_{t o t} / \delta g^{\mu \nu}=0$ yields the modified Einstein equations

$$
\begin{aligned}
(1-\alpha \varphi) R_{\mu \nu} & -\frac{1}{2}(1-\alpha \varphi) R g_{\mu \nu} \\
& +\alpha \nabla_{\mu} \nabla_{\nu} \varphi-\alpha g_{\mu \nu} \square \varphi-V g_{\mu \nu}=8 \pi G T_{\mu \nu}
\end{aligned}
$$

where $\square \equiv g^{\mu \nu} \nabla_{\mu} \nabla_{\nu}$ and we have introduced $\alpha \equiv 2 \gamma / M_{\mathrm{pl}}^{2}$. Variation with respect to the field $\varphi$ gives

$$
\gamma R=\frac{\partial V}{\partial \varphi}+\frac{1}{f_{0}} \frac{\partial f\left(\varphi / M_{\mathrm{pl}}\right)}{\partial \varphi} \rho_{M} .
$$

Equation (14) is the key to imposing the limiting curvature construction. It is clear that the curvature $R$ will remain bounded and approach a constant curvature at late times 
$(t \rightarrow \infty)$ as long as $V^{\prime}+f^{\prime} \rho_{M} \rightarrow$ constant; this is the essence of the construction. We assume a flat $(k=0)$ Friedmann-Robertson-Walker metric

$$
d s^{2}=-d t^{2}+a^{2}(t)\left\{d r^{2}+r^{2} d \Omega^{2}\right\},
$$

where $a(t)$ is the scale factor of the Universe and $d \Omega$ is the line-element of the unit 2 -sphere. Defining the Hubble parameter by $H \equiv \dot{a} / a$ and substituting the metric ansatz (15) into (13) and (14) gives the generalized Friedmann equation (the 00component of (13) ) and the equation of motion for $\varphi$ :

$$
\begin{aligned}
& 3 M_{\mathrm{pl}}^{2} H^{2}-6 \gamma \varphi H^{2}-6 \gamma H \dot{\varphi}+V(\varphi)=\frac{f\left(\varphi / M_{\mathrm{pl}}\right) \rho_{M}^{(0)}}{f_{0} a^{3}} \\
& 6 \gamma\left(2 H^{2}+\dot{H}\right)-V^{\prime}(\varphi)=f^{\prime}\left(\frac{\varphi}{M_{\mathrm{pl}}}\right) \frac{\rho_{M}^{(0)}}{f_{0} a^{3}}
\end{aligned}
$$

where a prime denotes differentiation with respect to $\varphi, f_{0} \equiv f\left(\varphi_{0} / M_{\mathrm{pl}}\right), \varphi_{0}$ and $\rho_{M}^{(0)}$ are the values of $\varphi$ and $\rho_{M}$ today.

By considering the asymptotics of our cosmology at early and late times, we can constrain the forms of the functions $V(\varphi)$ and $f(\varphi)$. We require that the effective Newton's constant for our theory remain positive definite so that gravity is always attractive. This imposes a constraint on $\Phi \geq 0, \forall t$ in equation (8) $)$. There are rather strong constrains on the time variation of Newton's constant from the period of nucleosynthesis until today (roughly, $|\dot{G} / G|<\mathcal{O}\left(10^{-10}-10^{-13} y^{-1}\right)$ [39]). For the time being, we will allow ourselves to ignore this constraint in order to produce a toy model capable of realizing the MC conjecture. Furthermore, because we are only interested in the behavior of the Universe from the matter dominated epoch until today, we have ignored the presence of radiation. The absolute earliest our theory is valid is up to the period of equal matter and radiation domination $t_{e q}$. For specificity, by early times we refer to times near the time of photon decoupling at a redshift of $z \simeq 1100$, during which the Universe is typically already well into the matter dominated regime.

At late times (and low curvatures) we want to bound the Ricci scalar $R$ from below. This will constitute a successful example of a model obeying the minimal curvature hypothesis. To bound the curvature we use equation (14). It is clear that the curvature $R$ will remain bounded if we bound $V^{\prime}+f^{\prime} \rho_{M}$, where the prime denotes differentiation with respect to $\varphi$. Hence, we require $\gamma^{-1}\left(V^{\prime}+f^{\prime} \rho_{M}\right) \rightarrow \mathcal{R}$ at late times, where we denote the hypothesized minimal curvature scale by $\mathcal{R}$. We anticipate that, by construction, there will be a late-time attractor that is a constant curvature space-time with $R=\mathcal{R}$. This attractor is not an actual solution to the equations of motion. The above considerations restrict the functional forms of potential $V$ and the non-minimal coupling function $f$. Integration singles out a class of theories that must obey $V(\varphi)+f(\varphi) \rho_{M} \rightarrow \gamma \mathcal{R} \varphi$ as $t \rightarrow \infty$. The simplest forms for $V$ and $f$ obeying the above constraint are the linear functions

$$
V(\varphi)=\mu^{3} \varphi, \quad f(\varphi)=\frac{\varphi}{M_{\mathrm{pl}}}
$$


where $\mu$ is, in principle, another free parameter with dimensions of mass. However, we will take $\mu=\gamma$, so that $\mathcal{R}=\gamma^{2}$, and $\gamma$ is the only free parameter in the theory $\amalg$. We now rescale time $t \equiv t H_{0}$ so that today $t_{0}=1$ and introduce the following dimensionless quantities

$$
\begin{aligned}
& a \equiv \frac{a}{a_{0}}, H \equiv \frac{H}{H_{0}} \varphi \equiv \frac{\varphi}{M_{p l}}, \gamma \equiv \frac{\gamma}{M_{p l}} \\
& \Omega_{M} \equiv \frac{\rho_{M}}{\rho_{c}}=\frac{\rho_{M}}{3 H^{2} M_{\mathrm{pl}}^{2}}, R=\frac{R}{H_{0}^{2}},
\end{aligned}
$$

and take $a_{0}=1$, where $a_{0}$ is the value of the scale factor today.

In terms of the dimensionless quantities the EOM become

$$
\begin{aligned}
& H^{2}-2 \gamma \varphi H^{2}-2 \gamma H \dot{\varphi}+\frac{V(\varphi)}{3}=\frac{f(\varphi) \Omega_{M}^{(0)}}{f_{0} a^{3}}, \\
& \gamma\left(2 H^{2}+\dot{H}\right)-\frac{V^{\prime}(\varphi)}{6}=f^{\prime}(\varphi) \frac{\Omega_{M}^{(0)}}{2 f_{0} a^{3}},
\end{aligned}
$$

The successful implementation of the minimal curvature hypothesis is now apparent. Recasting equation (21) in terms of the curvature scalar $R=6\left(2 H^{2}+\dot{H}\right)$, and substituting in our choices for $V$ and $f$ (17):

$$
R=\gamma^{2}+3 \frac{\Omega_{M}^{(0)}}{a^{3}}
$$

We see that, as the Universe expands and the matter term dilutes, we asymptotically approach the minimal value of the curvature $R=\mathcal{R}=\gamma^{2}$.

It is both interesting and surprising that the solution to Eq. (22) reduces to the simple case of $\Lambda$ CDM plus an arbitrary amount of dark radiation which may have either positive or negative effective energy density. Most notably, this model arises in RandallSundrum brane cosmology which has been extensively studied in the literature $\$$. The Friedmann equation derived from the Randall-Sundrum model for a flat Universe is

$$
H^{2}=\frac{\rho}{3 M_{\mathrm{pl}}^{2}}+\frac{\rho^{2}}{36 M_{5}^{6}}+\frac{\mathcal{C}}{a^{4}},
$$

where $M_{5}$ is the five dimensional Planck mass and $\mathcal{C} a^{-4}$ is the so called dark radiation term, since it scales like radiation, but it's origins are purely gravitational and it does not interact with standard matter [45]. At low energies (when the energy density is much less than the critical brane tension), the $\rho^{2}$ term can be safely neglected. The main observational restrictions on the dark radiation term come from the acoustic scale at recombination (see, e.g. [46]), and from the amount of total growth of density perturbations in the non-relativistic matter component from the time of equal matter and radiation until the present day [47, 48]. As a result, the density of dark radiation cannot be significantly larger than the present CMB energy density $\square$.

$\|$ It is interesting to note that if we do not take $\mu=\gamma$ and then take the limit that $\gamma \rightarrow 0$ the theory resembles the action of the modified source gravity models studied in 38 .

ๆ For some reviews see, e.g. 43, 44.

+ Note, the constraints from BBN are even stronger [49]. 
Making use of (18) and (19), Eq. (23) may be recast (neglecting the $\rho^{2}$ term) as

$$
H^{2}=\frac{\Omega_{M}}{a^{3}}+\frac{\Omega_{R}}{a^{4}}+\frac{\Omega_{\Lambda}}{3},
$$

where we have included a cosmological constant term $\Omega_{\Lambda}=\Lambda / 3 M_{\mathrm{pl}}^{2} H^{2}$, and $\Omega_{R}=$ $\Omega_{\mathcal{C}}+\Omega_{r}$ includes contributions from both the dark and ordinary radiation. From (24) we find

$$
\dot{H}=\frac{-3 \Omega_{M}}{2 a^{3}}-\frac{2 \Omega_{R}}{a^{4}} .
$$

Constructing the Ricci Scalar $R=6\left(2 H^{2}+\dot{H}\right)$ from the above expressions yields Eq. (22), with $\gamma^{2}=4 \Omega_{\Lambda}$.

In Appendix A, we provide a detailed analysis of the vacuum $\mathrm{MC}$ equations with $\Omega_{M}=0$. Although the presence of matter plays an important role in our minimal curvature construction, an analysis of the vacuum theory provides valuable insight into the solutions we are interested in studying. In Appendix B, we transform the vacuum $\mathrm{MC}$ theory into an Einstein frame and relate quantities of physical interest in both frames.

For general solutions to the equations of motion (20) and (21) with functions (17) we solve for the Hubble parameter $H(t)$ and scalar field $\varphi(t)$. We plot the relevant portion of the $\varphi-H$ phase space in Fig. 1.

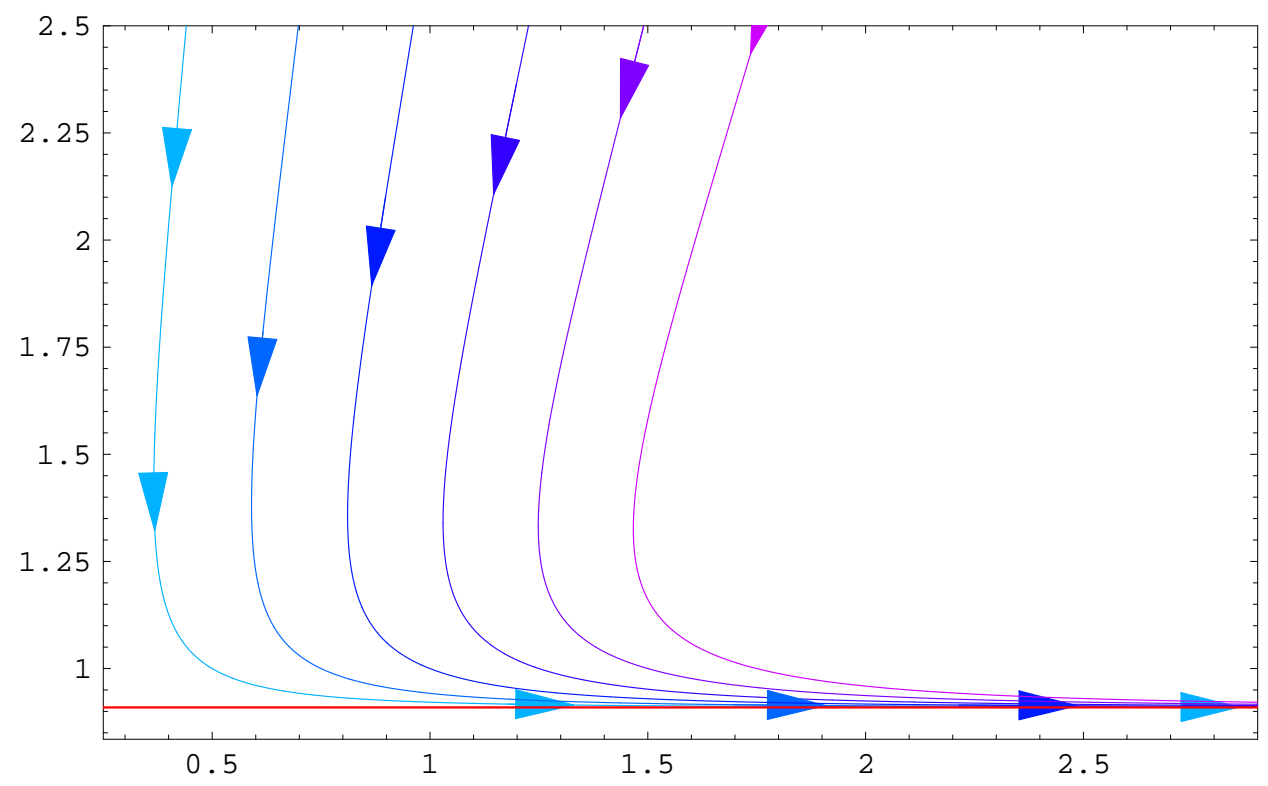

Figure 1. Phase space of $\varphi$ vs. $H$. All solutions asymptotically approach a late-time accelerating phase with constant $H$ at the minimal curvature $\mathcal{R}$ denoted by the red line.

To solve the equation we integrate from the past to today $\left(H_{0}=1\right)$ and then from today into the future and patch the solutions together. In the plots we take the conditions $H_{0}=1.0, \gamma=3.15, \Omega_{M}=0.25$ and let the values of $\varphi_{0}$ vary. At late times, the solutions approach the constant $H$ attractor when the Universe is well into the accelerating epoch. 


\section{Comparison with $\Lambda \mathrm{CDM}$}

We now compare our model with $\Lambda$ CDM. To do so, we must enter reasonable initial conditions into our numerical study and solve the equations of motion (201), (21) together with (17) and the equation $\dot{a}=H a$. Let us begin by considering the value of the minimal curvature. Physically the minimal curvature corresponds to an emptying of the matter in the Universe due to cosmological expansion. In our model the value of the minimal curvature is approached asymptotically. Today, the value of the curvature is given by

$$
R_{0}=6\left(2 H_{0}^{2}+\dot{H}_{0}\right) \approx 12 H_{0}^{2} .
$$

Because we observe a significant amount a matter in the Universe today, we know that we have not yet reached the minimal curvature. However, because we are accelerating, we know that we are near the minimal curvature (i.e. the first and second terms on the right hand side of equation (22) must be comparable). Hence, from equation (26), we expect the value of the minimal curvature $\mathcal{R}$ to be close to but less than $\mathcal{R}<12 H_{0}^{2}$. Therefore, in terms of our dimensionless quantities (18), $\mathcal{R}<12$ and the free parameter in our theory $\gamma<\sqrt{12} \approx 3.4641$. For the solutions considered below, we choose a value of $\gamma=3.15$ meeting the above requirements and that follows the $\Lambda$ CDM model to our satisfaction for our toy construction (i.e. a value leading to a matter dominated cosmology followed by a "jerk" near a redshift of $z \simeq .5$ into an accelerating phase).

In the action ([6), the effective Newton's constant is $G_{N_{e f f}}$ is given by

$$
\left(16 \pi G_{N_{e f f}}\right)^{-1}=\frac{M_{\mathrm{pl}}^{2}}{2}-\gamma \varphi
$$

To ensure that the effective Newton's constant remains positive definite over the history

of the Universe $\left(G_{N_{e f f}}>0, \forall t\right)$ we must have $\gamma \varphi<.5$. We are almost in a position to compare our model both with $\Lambda \mathrm{CDM}$, which has the Friedmann equation

$$
H=\sqrt{\frac{\Omega_{M}^{(0)}}{a^{3}}+\left(1-\Omega_{M}^{(0)}\right)}
$$

and with the observational data provided by the SNeIa gold sample. To make a comparison with the observational data we require an understanding of the luminosity distance formula in the context of modified gravity models.

An important consideration arises when using the formula for the luminosity distance in theories of the form:

$$
S=\int d^{4} x \sqrt{-g}\left[A(\varphi) \frac{R}{2}+B(\varphi)\left(\partial_{\mu} \varphi\right)^{2}+V(\varphi)+\sum_{i} C_{i}(\varphi) \mathcal{L}_{i}\right],
$$

where the $\mathcal{L}_{i}$ represent the different types of possible matter Lagrangians present. Such a theory arises as the low-energy effective action for the massless modes of dilaton gravity in string theory, and our model is an action of just this sort [41, 42]; albeit, with an unusual choice for the functions $A, B, V, C$. As we have already discussed, these theories typically lead to time variation in Newton's gravitational constant. The time variation can affect the way one should compare the theory to observations [50]. In 
particular, the time-evolution can alter the basic physics of supernovae. For example, the time variation in $G_{N}$ leads to different values of the Chandrasekhar mass at different epochs, and hence, a supernova's peak luminosity will vary depending on when the supernova exploded. This makes treating the supernovae Ia as standard candles difficult [51, 52, 53, 54, 55, 56. Specifically, the peak luminosity of SNeIa is proportional to the mass of nickel synthesized which is a fixed fraction of the Chandrasekhar mass $M_{C h} \sim G^{-3 / 2}$. Hence, the luminosity peak of SNeIa varies as $L \sim G^{-3 / 2}$ and the corresponding absolute magnitude evolves as

$$
M=M_{0}+\frac{15}{4} \log \frac{G(z)}{G_{0}},
$$

where the subscript zero indicates the local values of the quantities. Therefore, the magnitude-redshift relation of SNeIa in modified gravity theories of the type given by (29) is related to the luminosity distance via [53, 55]:

$$
m(z)=M_{0}+5 \log d_{L}(z)+\frac{15}{4} \log \frac{G(z)}{G_{0}} .
$$

Even if gravitational physics is described by some theory other than General Relativity the standard formula for the luminosity distance applies as long as one is considering a metric theory of gravity [57]:

$$
d_{L}\left(z ; H(z), H_{0}\right)=\frac{(1+z)}{H_{0}} \int_{0}^{z} \frac{d z^{\prime}}{H\left(z^{\prime}\right)} .
$$

For $\Lambda \mathrm{CDM}$, the Luminosity distance (32) can be written [40]:

$$
\begin{aligned}
& d_{L}\left(z ; \Omega_{M}, \Omega_{\Lambda}, H_{0}\right)= \\
& \frac{c(1+z)}{H_{0} \sqrt{|\kappa|}} \mathcal{S}\left(\sqrt{|\kappa|} \int_{0}^{z}\left[\left(1+z^{\prime}\right)^{2}\left(1+\Omega_{M} z^{\prime}\right)-z^{\prime}\left(2+z^{\prime}\right) \Omega_{\Lambda}\right]^{-\frac{1}{2}}\right)
\end{aligned}
$$

where, $\mathcal{S}(x) \equiv \sin (x)$ and $\kappa=1-\Omega_{\text {tot }}$ for $\Omega_{t o t}>1$ while $\mathcal{S}(x) \equiv \sinh (x)$ with $\kappa=1-\Omega_{\text {tot }}$ for $\Omega_{t o t}<1$ while $\mathcal{S}(x) \equiv x$ and $\kappa=1$, for $\Omega_{t o t}=1$. Here and throughout, $\Omega_{t o t}=\Omega_{M}+\Omega_{\Lambda}$.

\subsection{Numerical Analysis}

Given the above considerations we take the following values of parameters today:

$$
a_{0}=1.0, \quad H_{0}=1.0, \quad \varphi_{0}=0.1, \quad \gamma=3.15, \quad \Omega_{M}^{(0)}=0.25 .
$$

We then integrate our equations from the past to today and then from today into the future and patch the solutions together. While we do not provide an exhaustive study of the parameter space for the MC model, the parameters given above provide a successful example of the construction which fulfills our rather modest goals *. It is quite possible that the parameters (34) can be tuned to achieve an even better agreement with $\Lambda \mathrm{CDM}$.

\footnotetext{
* An exhaustive study of the phase space of the vacuum theory is supplied in Appendix A.
} 


\subsection{Results}

The history and future of the curvature $R$ together with the co-moving Hubble radius are plotted in Fig. 2,
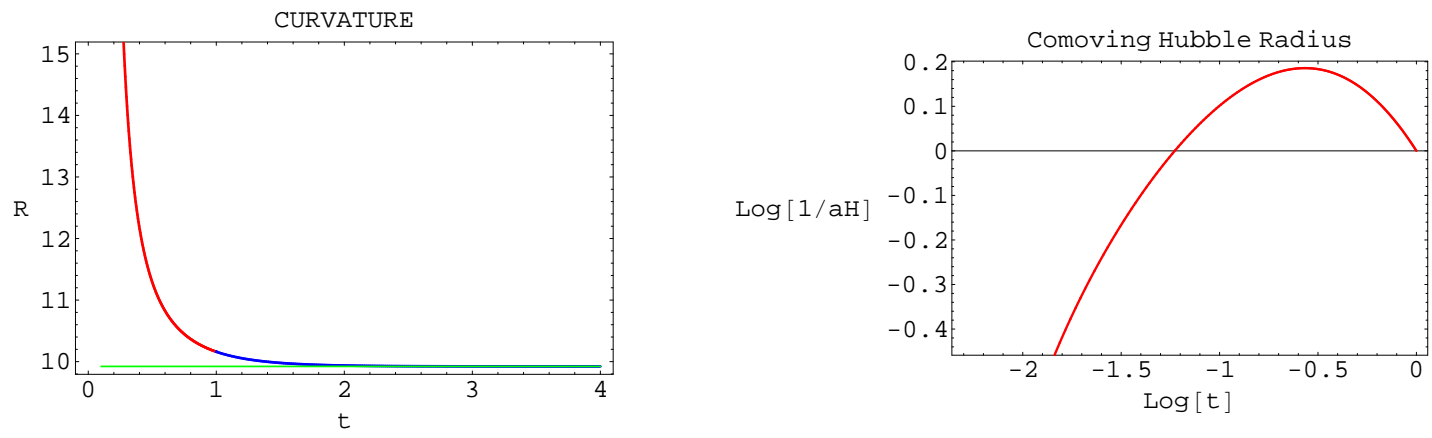

Figure 2. The Ricci scalar (left) and co-moving Hubble radius (right) as functions of cosmic time $t$. The curvature decreases from the matter-dominate past (red curve) to the constant minimal value at $R=\gamma^{2}$ indicated by the green line. Today, $t_{0}=1$ and the blue curve indicates the future evolution of the curvature. While the Universe is accelerating the co-moving Hubble radius decreases.

In the Figure the past history of the curvature is plotted in red while the future is plotted in blue. Today we are at the value $t=1$. The minimal curvature is given by the green line at $R=\mathcal{R}=\gamma^{2}$. As expected, the curvature is large in the past when the Universe is matter dominated and then decreases, approaching the accelerating late-time de-Sitter attractor with constant minimal curvature $R=\mathcal{R}$. The second plot in the Figure shows the evolution of the co-moving Hubble radius $H^{-1} / a$. The phenomenologically desired cosmological transition from matter domination to latetime acceleration of the Universe is clearly indicated by the decreasing of the co-moving Hubble radius, when

$$
\frac{d}{d t} \frac{H^{-1}}{a}<0 .
$$

In some modified gravity models it can be difficult to achieve this transition (see, e.g. [58, 59]).

The majority of our results are presented in Figures 3 and 4. In Figure 3 we plot the scale factor $a$ as a function of cosmic time $t$, the Hubble parameter $H$ as function of redshift $z$, where

$$
z+1 \equiv \frac{a_{0}}{a}
$$

the deceleration parameter

$$
q(z)=-\frac{\ddot{a}}{a H^{2}},
$$

and the luminosity distance, $d_{L}$. 

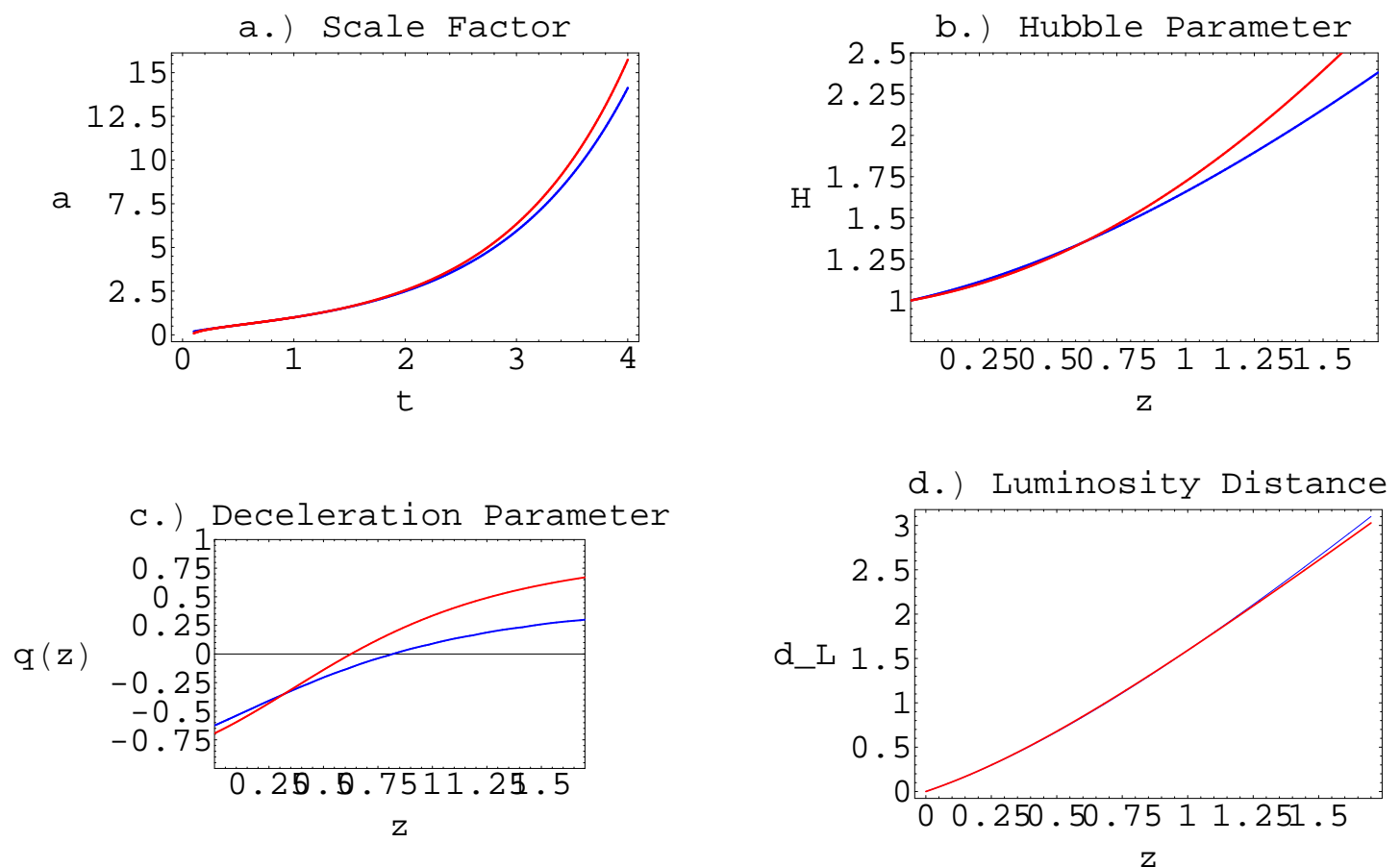

Figure 3. A comparison of the cosmological evolution of the MC model with $\Lambda$ CDM. Quantities plotted in red are the MC model while those in blue are for $\Lambda$ CDM. Today we are at the values $t=1, z=0$. Plot a.) shows the time evolution of the scale factor. Plot b.) shows the Hubble parameter as a function of redshift. Plot c.) compares the deceleration parameters as a function of redshift. Plot d.) shows the luminosity distance, $d_{L}$, as a function of redshift. The MC model is clearly a good fit with $\Lambda$ CDM.

For the particular set of parameters considered, the scale factor of our model differs from $\Lambda \mathrm{CDM}$ most strongly in the far future, although the difference in the expansion rates of the $\mathrm{MC}$ construction with $\Lambda \mathrm{CDM}$ is apparent in the plot of the Hubble parameters at high redshifts. The transition from matter domination to acceleration (the jerk) occurs at a slightly lower redshift than $\Lambda$ CDM. There is only a slight difference in $d_{L}$ that occurs at high redshifts $(z>1)$, although the difference is not significant enough to distinguish our model from $\Lambda \mathrm{CDM}$ using only the current supernova data. 


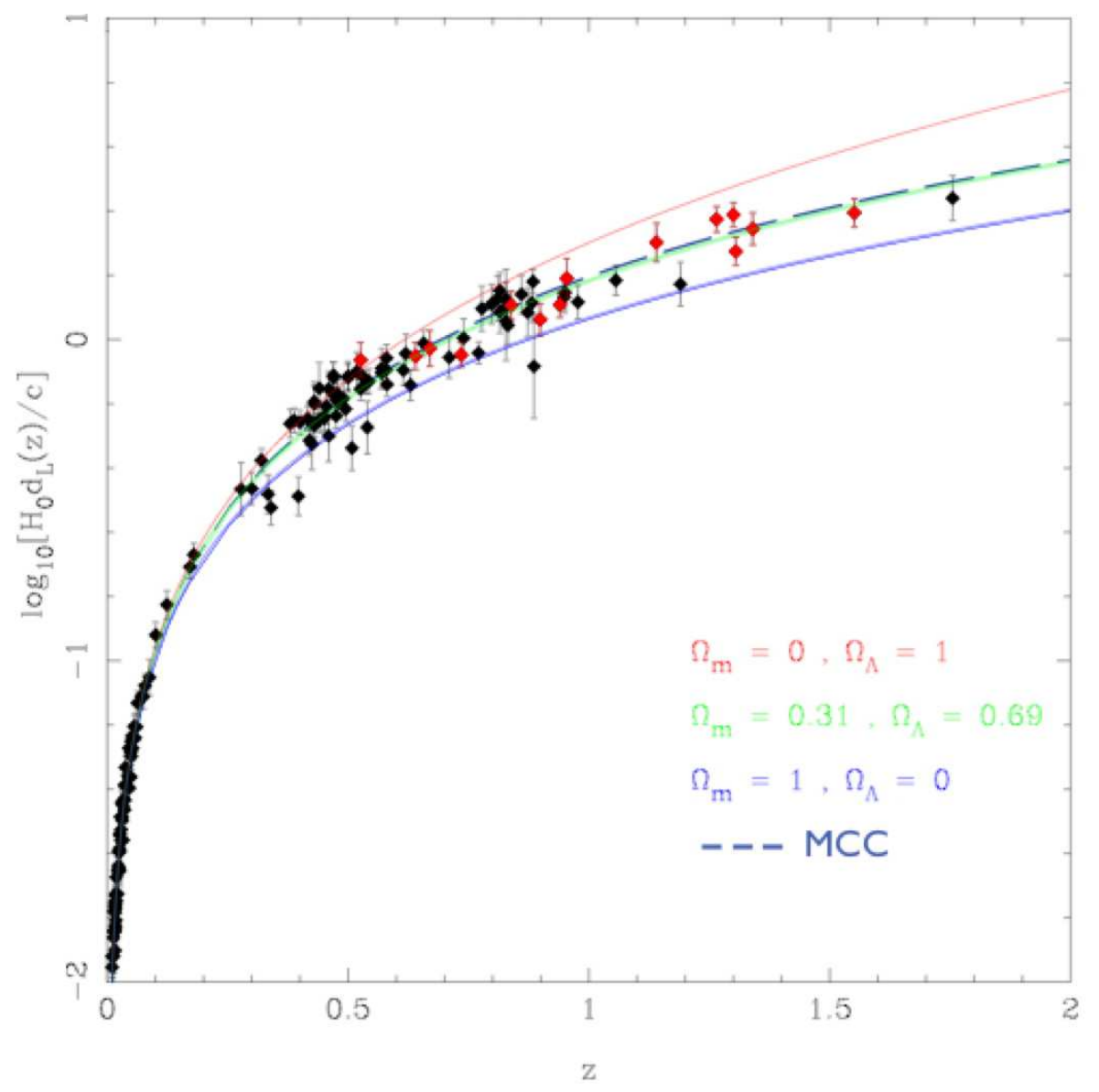

Figure 4. Comparison of the MC and various $\Lambda \mathrm{CDM}$ models with observational data. The supernovae data points are plotted with error bars and the data is taken from [27. The luminosity distance $d_{L}$ for the MC model is plotted by the dashed (blue) curve. The various theoretical predictions for $\Lambda \mathrm{CDM}$ are represented by the solid curves and were examined in 28 .

In Figure 4, we compare the luminosity distance predicted by our model with several versions of $\Lambda \mathrm{CDM}$ and with the observational data. The luminosity distances are plotted out to a redshift of $z=2$ (the highest redshift supernova data is from $z=1.76$ ). The theoretical predictions of the minimal curvature construction and $\Lambda$ CDM are compared with the "gold" supernovae sample of [27]. The particular choice of $\Lambda$ CDM models shown are from [28]. The luminosity distance for the minimal curvature construction is denoted by the blue dashed line and fits the supernova data extremely well. The luminosity distance $d_{L}$ is virtually indistinguishable from $\Lambda$ CDM with $\Omega_{M}^{(0)}=0.25$ and $\Omega_{\Lambda}^{(0)}=0.75$ (there is a small discrepancy at high redshift as can be seen from Figure 3).

\section{Conclusions}

We have shown that a period of late-time cosmic acceleration can follow directly from a simple minimal curvature conjecture (MCC). The model fits the SNeIa data exceptionally well. While the specific formulation considered here is only a toy 
construction, unlikely to be compatible with constraints from solar system and table top test of the equivalence principle, it may capture phenomenologically interesting features of a more fundamental theory that admits a limiting minimal curvature. Furthermore, the construction successfully demonstrates the possibility that a new fundamental physical principle may ultimately be responsible for the recent period of cosmological acceleration. It is possible that experimentally viable models based on the minimal curvature conjecture exist. The search for such models within the context of scalar-Gauss-Bonnet gravity is currently underway. Despite the tight theoretical and experimental constraints on scalar-Gauss-Bonnet cosmologies [62, 63, 64, 65, 66], we remain optimistic that an experimentally and theoretically viable model based on the minimal curvature construction can be discovered.

\section{Acknowledgments}

It is a pleasure to thank R. Brandenberger, R. Gregory, V. Jejjala, I. Moss, R. Myers and T. Underwood for helpful discussions. I am especially grateful to M. Trodden for numerous useful discussions over the course of this work. This work is supported in part by PPARC and by the EU 6th Framework Marie Curie Research and Training network "UniverseNet" (MRTN-CT-2006-035863).

\section{References}

[1] A. G. Riess et al. [Supernova Search Team Collaboration], Astron. J. 116, 1009 (1998); arXiv:astro-ph/9805201;

[2] S. Perlmutter et al. [Supernova Cosmology Project Collaboration], Astrophys. J. 517, 565 (1999); arXiv:astro-ph/9812133;

[3] C. B. Netterfield et al. [Boomerang Collaboration], Astrophys. J. 571, 604 (2002); arXiv:astro-ph/0104460;

[4] N. W. Halverson et al., Astrophys. J. 568, 38 (2002). arXiv:astro-ph/0104489.

[5] J. L. Tonry et al., Astrophys. J. 594, 1 (2003); arXiv:astro-ph/0305008;

[6] C. L. Bennett et al., Astrophys. J. Suppl. 148, 1 (2003); arXiv:astro-ph/0302207;

[7] S. Nojiri and S. D. Odintsov, arXiv:hep-th/0601213.

[8] E. J. Copeland, M. Sami and S. Tsujikawa, Int. J. Mod. Phys. D 15, 1753 (2006) arXiv:hep-th/0603057.

[9] S. M. Carroll, V. Duvvuri, M. Trodden and M. S. Turner, Phys. Rev. D 70, 043528 (2004) arXiv:astro-ph/0306438.

[10] S. M. Carroll, A. De Felice, V. Duvvuri, D. A. Easson, M. Trodden and M. S. Turner, Phys. Rev. D 71, 063513 (2005) arXiv:astro-ph/0410031.

[11] D. A. Easson, Int. J. Mod. Phys. A 19, 5343 (2004) arXiv:astro-ph/0411209.

[12] S. Nojiri and S. D. Odintsov, Mod. Phys. Lett. A 19, 627 (2004) arXiv:hep-th/0310045.

[13] F. P. Schuller and M. N. R. Wohlfarth, Phys. Lett. B 612, 93 (2005) arXiv:gr-qc/0411076.

[14] F. P. Schuller and M. N. R. Wohlfarth, Nucl. Phys. B 698, 319 (2004) arXiv:hep-th/0403056.

[15] M. A. Markov, Pisma Zh. Eksp. Teor. Fiz. 36, 214 (1982); Pisma Zh. Eksp. Teor. Fiz. 46, 342 (1987); V. P. Frolov, M. A. Markov and V. F. Mukhanov, Phys. Lett. B 216, 272 (1989).

[16] V. P. Frolov, M. A. Markov and V. F. Mukhanov, Phys. Rev. D 41, 383 (1990).

[17] D. Morgan, Phys. Rev. D 43, 3144 (1991).

[18] V. Mukhanov and R. H. Brandenberger, Phys. Rev. Lett. 68, 1969 (1992);

[19] M. Trodden, V. F. Mukhanov and R. H. Brandenberger, Phys. Lett. B 316, 483 (1993); arXiv:hep-th/9305111;

[20] R. H. Brandenberger, V. Mukhanov and A. Sornborger, Phys. Rev. D 48, 1629 (1993); arXiv:gr-qc/9303001. 
[21] R. Moessner and M. Trodden, Phys. Rev. D 51, 2801 (1995); arXiv:gr-qc/9405004.

[22] R. H. Brandenberger, R. Easther and J. Maia, JHEP 9808, 007 (1998); arXiv:gr-qc/9806111.

[23] D. A. Easson and R. H. Brandenberger, JHEP 9909, 003 (1999); arXiv:hep-th/9905175.

[24] D. A. Easson and R. H. Brandenberger, JHEP 0106, 024 (2001); arXiv:hep-th/0103019.

[25] D. A. Easson, JHEP 0302, 037 (2003); arXiv:hep-th/0210016;

[26] D. A. Easson, Phys. Rev. D 68, 043514 (2003). arXiv:hep-th/0304168.

[27] A. G. Riess et al. [Supernova Search Team Collaboration], Astrophys. J. 607, 665 (2004) arXiv:astro-ph/0402512.

[28] T. R. Choudhury and T. Padmanabhan, Astron. Astrophys. 429, 807 (2005) arXiv:astro-ph/0311622.

[29] G. Magnano and L. M. Sokolowski, Phys. Rev. D 50, 5039 (1994) arXiv:gr-qc/9312008.

[30] T. Damour, G. W. Gibbons and C. Gundlach, Phys. Rev. Lett. 64, 123 (1990).

[31] R. Bean and J. Magueijo, Phys. Lett. B 517, 177 (2001) arXiv:astro-ph/0007199.

[32] R. Bean, Phys. Rev. D 64, 123516 (2001) arXiv:astro-ph/0104464.

[33] T. Biswas and A. Mazumdar, arXiv:hep-th/0408026.

[34] T. Biswas, R. Brandenberger, A. Mazumdar and T. Multamaki, Phys. Rev. D 74, 063501 (2006) arXiv:hep-th/0507199.

[35] R. Bean, S. Carroll and M. Trodden, arXiv:astro-ph/0510059.

[36] S. Das, P. S. Corasaniti and J. Khoury, Phys. Rev. D 73, 083509 (2006) arXiv:astro-ph/0510628.

[37] L. Amendola, D. Polarski and S. Tsujikawa, arXiv:astro-ph/0603703

[38] S. M. Carroll, I. Sawicki, A. Silvestri and M. Trodden, arXiv:astro-ph/0607458

[39] J. P. Uzan, Rev. Mod. Phys. 75, 403 (2003) [arXiv:hep-ph/0205340.

[40] S. Perlmutter et al. [Supernova Cosmology Project Collaboration], Astrophys. J. 483, 565 (1997) arXiv:astro-ph/9608192.

[41] T. Damour and A. M. Polyakov, Gen. Rel. Grav. 26, 1171 (1994) arXiv:gr-qc/9411069.

[42] T. Damour and A. M. Polyakov, Nucl. Phys. B 423, 532 (1994) arXiv:hep-th/9401069.

[43] D. A. Easson, Int. J. Mod. Phys. A 16, 4803 (2001) arXiv:hep-th/0003086.

[44] D. Langlois, Prog. Theor. Phys. Suppl. 148, 181 (2003) arXiv:hep-th/0209261].

[45] P. Binetruy, C. Deffayet, U. Ellwanger and D. Langlois, Phys. Lett. B 477, 285 (2000) arXiv:hep-th/9910219.

[46] Y. Wang and P. Mukherjee, Astrophys. J. 650, 1 (2006) arXiv:astro-ph/0604051.

[47] M. Doran and G. Robbers, JCAP 0606, 026 (2006) arXiv:astro-ph/0601544.

[48] E. V. Linder, Astropart. Phys. 26, 16 (2006) arXiv:astro-ph/0603584.

[49] D. Langlois, Prog. Theor. Phys. Suppl. 163, 258 (2006) arXiv:hep-th/0509231].

[50] V. Acquaviva, C. Baccigalupi, S. M. Leach, A. R. Liddle and F. Perrotta, Phys. Rev. D 71, 104025 (2005) arXiv:astro-ph/0412052.

[51] L. Amendola, P. S. Corasaniti and F. Occhionero, arXiv:astro-ph/9907222.

[52] E. Garcia-Berro, E. Gaztanaga, J. Isern, O. Benvenuto and L. Althaus, arXiv:astro-ph/9907440

[53] A. Riazuelo and J. P. Uzan, Phys. Rev. D 66, 023525 (2002) arXiv:astro-ph/0107386.

[54] E. Gaztanaga, E. Garcia-Berro, J. Isern, E. Bravo and I. Dominguez, Phys. Rev. D 65, 023506 (2002) arXiv:astro-ph/0109299.

[55] S. Nesseris and L. Perivolaropoulos, Phys. Rev. D 73, 103511 (2006) arXiv:astro-ph/0602053.

[56] R. Gannouji, D. Polarski, A. Ranquet and A. A. Starobinsky, JCAP 0609, 016 (2006) arXiv:astro-ph/0606287.

[57] C. Shapiro and M. S. Turner, arXiv:astro-ph/0512586.

[58] D. A. Easson, F. P. Schuller, M. Trodden and M. N. R. Wohlfarth, Phys. Rev. D 72, 043504 (2005) arXiv:astro-ph/0506392.

[59] R. Punzi, F. P. Schuller and M. N. R. Wohlfarth, arXiv:gr-qc/0605017.

[60] H. K. Jassal, J. S. Bagla and T. Padmanabhan, Phys. Rev. D 72, 103503 (2005) arXiv:astro-ph/0506748.

[61] H. K. Jassal, J. S. Bagla and T. Padmanabhan, arXiv:astro-ph/0601389.

[62] S. Nojiri and S. D. Odintsov, Phys. Lett. B 631, 1 (2005) arXiv:hep-th/0508049].

[63] G. Cognola, E. Elizalde, S. Nojiri, S. D. Odintsov and S. Zerbini, Phys. Rev. D 73, 084007 (2006) arXiv:hep-th/0601008.

[64] A. De Felice, M. Hindmarsh and M. Trodden, JCAP 0608, 005 (2006) arXiv:astro-ph/0604154.

[65] G. Calcagni, B. de Carlos and A. De Felice, Nucl. Phys. B 752, 404 (2006) arXiv:hep-th/0604201].

[66] T. Koivisto and D. F. Mota, Phys. Lett. B 644, 104 (2007) arXiv:astro-ph/0606078. 


\section{Appendix A. Probing the Vacuum Theory}

The basic mechanism used to implement the limiting curvature construction requires a non-minimal coupling of $\varphi$ to the matter Lagrangian. This coupling plays an important role in the overall cosmological dynamics discussed above. Hence, an understanding of the vacuum theory is perhaps not as fruitful as it would be in case of a theory with minimal coupling of $\varphi$ to matter; however, once the Universe becomes sufficiently large and dilute the dynamics will resemble those of the vacuum theory. As we shall see the vacuum $\mathrm{MC}$ theory is significantly richer than that of $\Lambda \mathrm{CDM}$. The equations describing the vacuum are given by the equations (16) with $\Omega_{M}=0$. The dimensionless forms are

$$
\begin{aligned}
& H^{2}-2 \gamma \varphi H^{2}-2 \gamma H \dot{\varphi}+\frac{V(\varphi)}{3}=0, \\
& \gamma\left(2 H^{2}+\dot{H}\right)-\frac{V^{\prime}(\varphi)}{6}=0,
\end{aligned}
$$

where we have re-introduced the parameter $\mu$ from equation (17) (previously set equal to $\gamma$ ) for completeness and the potential is $V(\varphi)=\mu^{3} \varphi$. In the case of the vacuum we may solve exactly for $H(t)$. We focus on two types of solutions of particular interest. In the first, the Hubble parameter is given by

$$
H(t)=\sqrt{\frac{1}{12} \mathcal{R}} \tanh \left\{\sqrt{\frac{\mathcal{R}}{3}}(t-6 \alpha \gamma)\right\},
$$

where $\mathcal{R}=\mu^{3} / \gamma$ is the minimal curvature and $\alpha$ is a constant. In this case

$$
\dot{H}=\frac{\mathcal{R}}{6} \cosh \left\{\sqrt{\frac{\mathcal{R}}{3}}(t-6 \alpha \gamma)\right\},
$$

and the scale factor evolves as

$$
a(t)=a_{0}\left[\operatorname{sech}\left\{\sqrt{\frac{\mathcal{R}}{3}}(6 \alpha \gamma-t)\right\}\right]^{\frac{1}{2}} .
$$

The quantities mentioned above, along with the field $\varphi$ are plotted in Fig. (A1). In this case the Universe undergoes a bounce as indicated in plots a.) and b.) in Fig. (A1). After the bounce the Hubble parameter is pulled up to the value at the minimum curvature $H \rightarrow \sqrt{\mathcal{R} / 12}$. This is a de Sitter solution of the vacuum theory with

$$
H_{d S}=\sqrt{\mathcal{R} / 12}
$$

while $\varphi$ continues to evolve exponentially $\varphi(t)+(2 \gamma)^{-1} \propto \exp (\sqrt{\mathcal{R} / 12})$. Interestingly, $H$ and $\dot{H}$ evolve in such a way that the curvature $R=6(2 H+\dot{H})$ is constant throughout the evolution of the solution, fixed at the minimal value $R=\mathcal{R}$. This behavior is not surprising as the constraint on $R$ comes from the second equation in the EOM (A.1).

The second family of solutions are of greater relevance. They are the vacuum analogs of the solutions plotted in Fig. (1) and discussed at the end of Section 2. These solution are pulled down to the value of $H$ at the minimal curvature. The entire phase space of solutions to the vacuum theory is shown in Fig. (A2). Representative solutions 

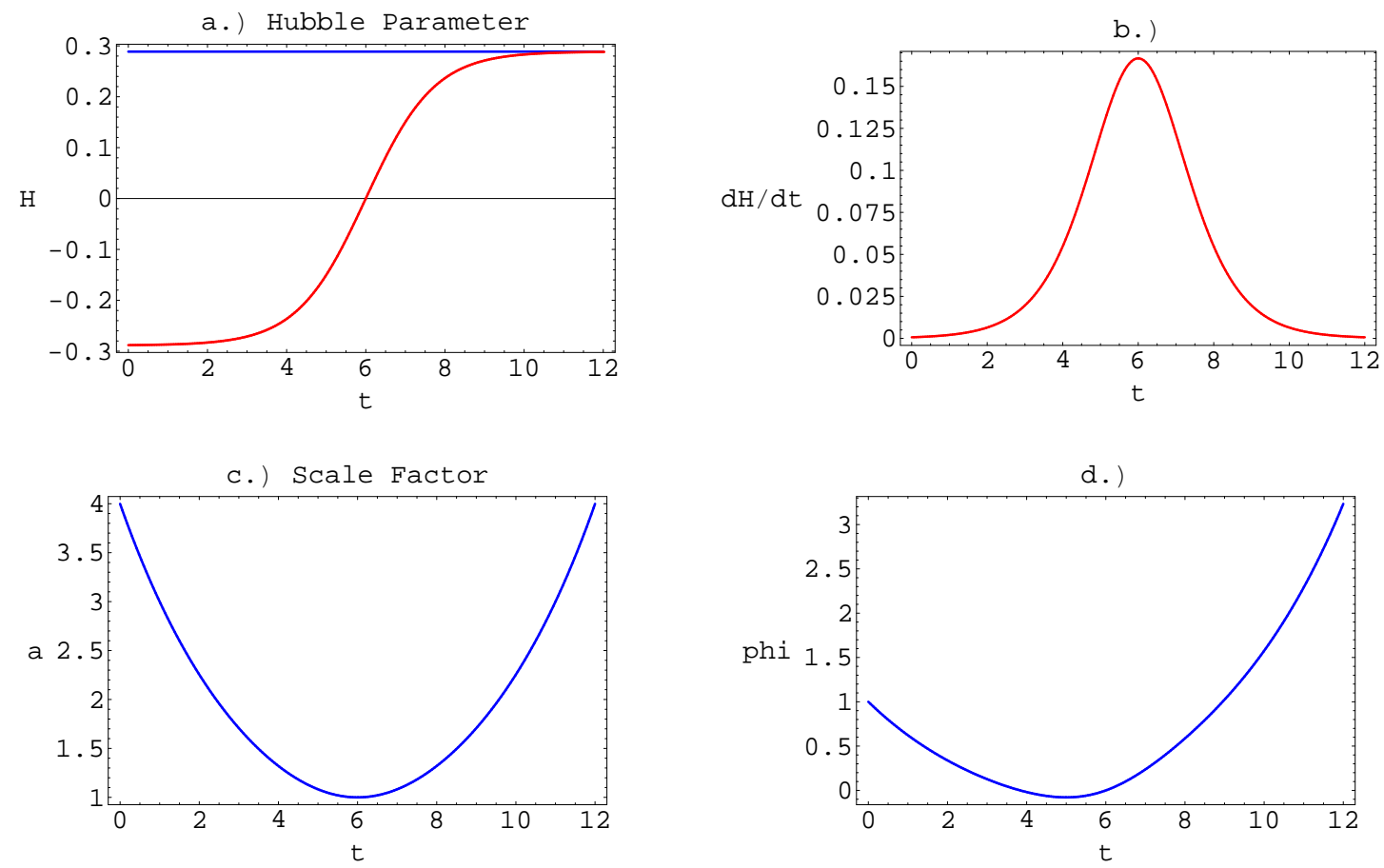

Figure A1. Various quantities of interested plotted as functions of cosmic time $t$. In the plots we take $\mathcal{R}=\gamma=\mu=\alpha=1$.

of the two families of solutions discussed above are plotted by the solid curves. The red curve is the solution given by equation (A.2). The second set of solutions (relevant to Section 21) are plotted in green in the upper right quadrant of the phase space. The system defined by the vacuum equations (equation (A.1)) has two unstable saddle equilibrium points at the values

$$
(\varphi, H)=\left(-\frac{M_{\mathrm{pl}}^{2}}{2 \gamma}, \pm \sqrt{\frac{\mathcal{R}}{12}}\right) .
$$

The equilibrium points are marked by the purple dots in Fig. (A2). 


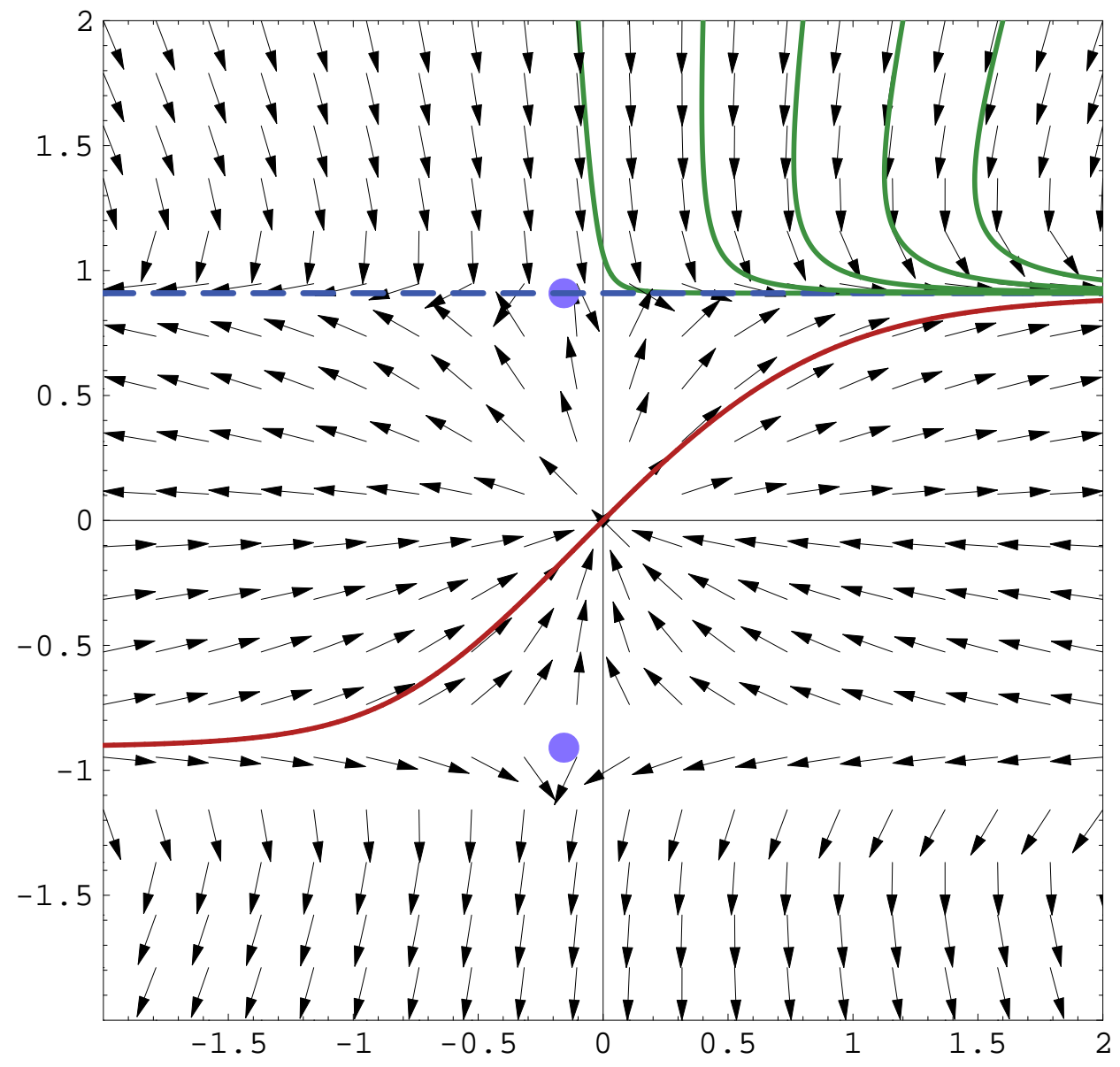

Figure A2. The phase space of the vacuum theory. $H$ is plotted on the vertical axis and $\varphi$ is plotted on the horizontal axis. The vector field for solutions is drawn in black. Solutions of particular interests are plotted by the solid curves. The constant de Sitter value of $H$ at the minimal curvature is indicated by the dashed (blue) line. The two unstable saddle equilibrium points are designated by the purple dots. In the plot we take $\gamma=\mu=3.15$.

\section{Appendix B. An Einstein Frame Description}

It is not our intent to provide a complete Einstein frame analysis of the full MC system with matter; however, it is instructive to consider the vacuum theory in an Einstein frame. To move to the Einstein frame we begin with the Brans-Dicke frame defined by the action (8). Passage to the Einstein frame is achieved via a conformal transformation of the form

$$
\tilde{g}_{\mu \nu}=\Omega^{2} g_{\mu \nu},
$$

where $\Omega^{2}$ is the conformal factor which must be positive to leave the signature of the metric unaltered. From this point forward a tilde shall denote a quantity built out of the Einstein-frame metric tensor $\tilde{g}_{\mu \nu}$. Under this transformation the infinitesimal line 
element and the determinant of the metric transform as

$$
d \tilde{s}^{2}=\Omega^{2} d s^{2}, \quad \sqrt{-\tilde{g}}=\Omega^{4} \sqrt{-g},
$$

respectively. The Ricci scalar transforms as

$$
R=\Omega^{2}\left(\tilde{R}+6 \widetilde{\square}(\ln \Omega)-6 \tilde{g}^{\mu \nu} \frac{\widetilde{\nabla}_{\mu} \Omega \widetilde{\nabla}_{\nu} \Omega}{\Omega^{2}}\right) .
$$

Omitting the ordinary divergence $\sqrt{-\tilde{g}} \widetilde{\square}(\ln \Omega)$, the action in the Brans-Dicke frame transforms to

$$
S=\int d^{4} x \sqrt{-\tilde{g}}\left(\frac{\Phi}{\Omega^{2}} \tilde{R}-6 \frac{\Phi}{\Omega^{4}} \tilde{g}^{\mu \nu} \widetilde{\nabla}_{\mu} \Omega \widetilde{\nabla}_{\nu} \Omega+\frac{V(\Phi)}{\Omega^{4}}\right),
$$

where the potential in terms of $\Phi$ is given by (7) together with (17):

$$
V(\Phi)=\frac{\mu^{3}}{\gamma}\left(\frac{M_{\mathrm{pl}}^{2}}{2}-\Phi\right) \text {. }
$$

Here we have re-introduced the parameter $\mu$ from equation (17) (previously set equal to $\gamma$ ) for completeness.

By choosing our conformal factor to be $\Omega^{2}=2 \Phi / M_{\mathrm{pl}}^{2}$, and performing a field redefinition to define the Einstein frame field $\phi$,

$$
\Phi=\frac{M_{\mathrm{pl}}^{2}}{2} \exp \left\{\sqrt{\frac{2}{3}} \frac{\phi}{M_{\mathrm{pl}}}\right\},
$$

the action (B.4) becomes the Einstein frame action

$$
S_{E F}=\int d^{4} x \sqrt{-\tilde{g}}\left(\frac{M_{\mathrm{pl}}^{2}}{2} \tilde{R}-\frac{1}{2} \tilde{g}^{\mu \nu} \widetilde{\nabla}_{\mu} \phi \widetilde{\nabla}_{\nu} \phi-\widetilde{V}(\phi)\right),
$$

where we have defined the potential

$$
\tilde{V}(\Phi)=-\left(\frac{M_{\mathrm{pl}}^{2}}{2}\right)^{2} \frac{V(\Phi)}{\Phi^{2}} .
$$

The Einstein frame potential is given by

$$
\tilde{V}(\phi)=\frac{M_{\mathrm{pl}}^{2}}{2} \mathcal{R}\left(e^{\beta \phi / M_{\mathrm{pl}}}-1\right) e^{-2 \beta \phi / M_{\mathrm{pl}}}
$$

where $\mathcal{R}=\mu^{3} / \gamma$ is the minimal curvature and $\beta=\sqrt{2 / 3}$.

In this frame the field $\phi$ has a canonically normalized kinetic term making the interpretation of solutions more simple due to our familiarity with minimally coupled scalar field to ordinary Einstein gravity. It is important to note, however, that so far we have only considered the vacuum theory. Even in the Einstein frame the Einstein field $\phi$ will couple non-minimally to matter and therefore, when matter is present in significant amounts, the simple Einstein frame vacuum solutions will not be an accurate description of the theory.

Under the conformal transformation (B.1), the cosmic time coordinate transforms as

$$
d \tilde{t}^{2}=e^{\beta \phi / M_{\mathrm{pl}}} d t^{2}
$$


Taking the Einstein-frame Friedmann-Robertson-Walker flat metric

$$
d \tilde{s}^{2}=-d \tilde{t}^{2}+\tilde{a}^{2}(\tilde{t}) d \mathbf{x}^{2},
$$

Leads to the familiar equations of motion

$$
\tilde{H}^{2}=\frac{1}{3 M_{\mathrm{pl}}^{2}}\left(\frac{1}{2} \phi^{2}+\tilde{V}(\phi)\right)
$$

and

$$
\phi^{\prime \prime}+3 \tilde{H} \phi^{\prime}+\frac{d \tilde{V}}{d \phi}=0,
$$

where prime denotes differentiation with respect to the Einstein frame cosmic time coordinate $\tilde{t}$ and $\tilde{H} \equiv \tilde{a}^{\prime} / \tilde{a}$. Using the conformal transformation (B.1), we find the Hubble parameters in the Einstein and Minimal Curvature frames are related by

$$
H=e^{\frac{\beta \phi}{2 M_{\mathrm{pl}}}}\left(\tilde{H}-\frac{\beta}{2 M_{\mathrm{pl}}} \phi^{\prime}\right) .
$$

We plot the Einstein frame potential (B.9) in Fig. (B1). From the plot it is clear

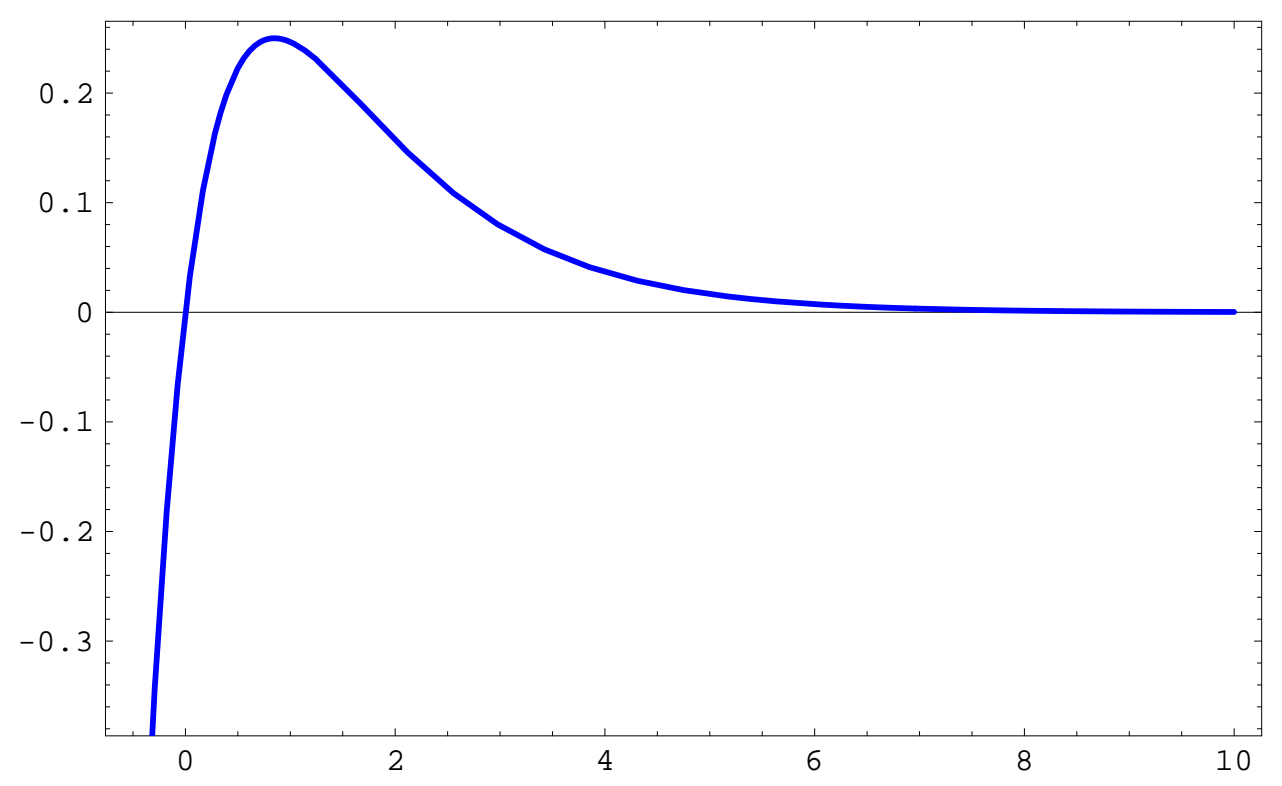

Figure B1. The Einstein frame potential $\widetilde{V}$ as a function of the Einstein frame field $\phi$. In the plot we have set $16 \pi G_{N}=\gamma=\mu=1$.

that there are three regions of interest:

i) There is an unstable de Sitter solution corresponding to the field sitting on the top of the potential.

ii) If the field starts initially to the left of the unstable de Sitter solution (and without significant positive velocity) the system will quickly run off to large negative $\phi$.

iii) If the field falls to the right of the de Sitter solution it will asymptotically roll to large positive values. 
Let us examine the first possibility in greater detail. The unstable de Sitter solution with constant $\tilde{H}=\tilde{H}_{d S}$ sits at the value at the maximum of the potential (B.9). The maximum is at constant value

$$
\phi=\phi_{d S}=\beta^{-1} M_{\mathrm{pl}} \ln 2,
$$

From equation (B.12) we find the corresponding $\tilde{H}$

$$
\tilde{H}_{d S}=\frac{1}{2} \sqrt{\frac{\mathcal{R}}{6}} .
$$

From equation (B.14) we see that the corresponding value in the minimal curvature frame is

$$
H\left(\tilde{H}_{d S}, \phi_{d S}\right)=\sqrt{\frac{\mathcal{R}}{12}},
$$

which is the exact de Sitter solution we found in our analysis of the vacuum of the minimal curvature theory (A.5).

The Minimal Curvature frame field is related to the Einstein frame field via

$$
\varphi(\phi)=\frac{M_{\mathrm{pl}}^{2}}{2 \gamma}\left(1-e^{\beta \phi / M_{\mathrm{pl}}}\right),
$$

and consequently,

$$
\phi(\varphi)=\frac{M_{\mathrm{pl}}}{\beta} \ln \left(1-\frac{2 \gamma}{M_{\mathrm{pl}}^{2}} \varphi\right) .
$$

Using the relation $(\underline{\mathrm{B} .18})$, we find the value of $\varphi$ at the de Sitter point $(\underline{\mathrm{B} .15})$ :

$$
\varphi\left(\phi_{d S}\right)=-\frac{M_{\mathrm{pl}}^{2}}{2 \gamma} .
$$

Hence we conclude that the unstable de Sitter solution in the Einstein frame is mapped to one of the unstable saddle critical points in the vacuum of the Minimal Curvature frame (see equation (A.6)).

We now examine case iii): when $\phi$ rolls to large positive values the Einstein frame potential may be approximated by

$$
\tilde{V}(\phi) \simeq \frac{M_{\mathrm{pl}}^{2} \mathcal{R}}{2} e^{-\beta \phi / M_{\mathrm{pl}}}
$$

leading to the exact solutions

$$
\begin{aligned}
& \frac{\phi(\tilde{t})}{M_{\mathrm{pl}}}=\sqrt{6} \ln \left(\sqrt{\frac{\tilde{V}_{0}}{24}} \frac{\tilde{t}}{M_{\mathrm{pl}}}\right) \\
& \tilde{H}(\tilde{t})=\frac{3}{\tilde{t}},
\end{aligned}
$$

where $\widetilde{V_{0}}=M_{\mathrm{pl}}^{2} \mathcal{R} / 2$, corresponding to power-law acceleration in the Einstein frame with

$$
\tilde{a}(\tilde{t})=\tilde{a}_{0} \tilde{t}^{3} .
$$

To find the corresponding behavior in the Minimimal Curvature frame we use the conformal transformation (B.1) along with the transformation for the cosmic time 
coordinate $(\bar{B} .10)$. We find the late-time power law attractor in the Einstein frame $(\overline{B .24})$ is mapped to the asymptotic de Sitter attractor in the MC frame at the minimal curvature $\mathcal{R}$ (depicted by the dashed blue line in Fig. (A2)),

$$
a(t)=a_{0} e^{\sqrt{\frac{\mathcal{R}}{12}} t} .
$$

